\title{
The role of Zur-regulated lipoprotein A in bacterial morphology, antimicrobial susceptibility, and production of outer membrane vesicles in Acinetobacter baumannii
}

\author{
Nayeong Kim ${ }^{1 \dagger}$, Hyo Jeong Kim ${ }^{1 \dagger}$, Man Hwan Oh², Se Yeon Kim', Mi Hyun Kim¹, Joo Hee Son', Seung II Kim³,
} Minsang Shin', Yoo Chul Lee ${ }^{1}$ and Je Chul Lee ${ }^{1 *}$ (D)

\begin{abstract}
Background: Zinc uptake-regulator (Zur)-regulated lipoprotein A (ZrIA) plays a role in bacterial fitness and overcoming antimicrobial exposure in Acinetobacter baumannii. This study further characterized the zrlA gene and its encoded protein and investigated the roles of the zrlA gene in bacterial morphology, antimicrobial susceptibility, and production of outer membrane vesicles (OMVs) in A. baumannii ATCC 17978.

Results: In silico and polymerase chain reaction analyses showed that the $z r / A$ gene was conserved among $A$. baumannii strains with 97-100\% sequence homology. Recombinant ZrlA protein exhibited a specific enzymatic activity of D-alanine-D-alanine carboxypeptidase. Wild-type A. baumannii exhibited more morphological heterogeneity than a $\Delta z r / A$ mutant strain during stationary phase. The $\Delta z r / A$ mutant strain was more susceptible to gentamicin than the wild-type strain. Sizes and protein profiles of OMVs were similar between the wild-type and $\Delta z r / A$ mutant strains, but the $\triangle$ zrlA mutant strain produced 9.7 times more OMV particles than the wild-type strain. OMVs from the $\triangle$ zrlA mutant were more cytotoxic in cultured epithelial cells than OMVs from the wild-type strain.

Conclusions: The present study demonstrated that A. baumannii ZrlA contributes to bacterial morphogenesis and antimicrobial resistance, but its deletion increases OMV production and OMV-mediated host cell cytotoxicity.
\end{abstract}

Keywords: Acinetobacter baumannii, Zur-regulated gene, ZrIA, Carboxypeptidase, Outer membrane vesicle

\section{Background}

Acinetobacter baumannii is a leading cause of nosocomial infections, including ventilator-associated pneumonia, skin and soft tissue infections, urinary tract infections, meningitis, and sepsis, particularly in intensive care units [1,2].

\footnotetext{
* Correspondence: leejc@knu.ac.kr

${ }^{\dagger}$ Nayeong Kim and Hyo Jeong Kim contributed equally to this work. 'Department of Microbiology, School of Medicine, Kyungpook National University, 680 Gukchaebosang-ro, Jung-gu, Daegu 41944, Republic of Korea Full list of author information is available at the end of the article
}

A. baumannii is a member of the 'ESKAPE' pathogens that are potentially drug-resistant bacteria [3]. The World Health Organization listed carbapenem-resistant $A$. baumannii as the most critical pathogen for development of new therapeutic agents. Like other pathogens, A. baumannii acquires nutrient metals, including iron, zinc (Zn), copper, magnesium, nickel, and manganese, from the host for a variety of biological processes [4-6]. However, hosts can limit the availability of these metals in a process referred to as nutritional immunity [4]. The acquisition of

(c) The Author(s). 2021 Open Access This article is licensed under a Creative Commons Attribution 4.0 International License, which permits use, sharing, adaptation, distribution and reproduction in any medium or format, as long as you give appropriate credit to the original author(s) and the source, provide a link to the Creative Commons licence, and indicate if changes were made. The images or other third party material in this article are included in the article's Creative Commons licence, unless indicated otherwise in a credit line to the material. If material is not included in the article's Creative Commons licence and your intended use is not permitted by statutory regulation or exceeds the permitted use, you will need to obtain permission directly from the copyright holder. To view a copy of this licence, visit http://creativecommons.org/licenses/by/4.0/ The Creative Commons Public Domain Dedication waiver (http://creativecommons.org/publicdomain/zero/1.0/) applies to the data made available in this article, unless otherwise stated in a credit line to the data. 
$\mathrm{Zn}$ and its utilization are associated with pathogenesis in A. baumannii [7]. The $\mathrm{Zn}$ uptake-regulator (Zur) is a conserved repressor that controls the expression of Zur-regulated genes. Mortensen et al. [7] identified 144 genes that were significantly up-regulated or downregulated in expression in the $\Delta z u r:: \mathrm{Km}$ mutant compared to that in A. baumannii ATCC 17978 using RNA-sequencing analysis [7]. The A1S_3412 gene encoding Zur-regulated lipoprotein A (ZrlA) is a significantly up-regulated (18.8-fold) during $\mathrm{Zn}$ starvation [7]. An $\triangle z r l A$ mutant exhibits increased envelope permeability and decreased membrane barrier function, which subsequently increases susceptibility to antimicrobial agents [8]. Moreover, this mutant strain exhibits reduced biofilm formation and surface motility, low adherence to epithelial cells, and low bacterial burden in the bloodstream compared to wild-type [9]. Thus, ZrlA contributes to antimicrobial resistance and pathogenicity in A. baumannii and is a potential target for anti-virulence agents against multidrug-resistant $A$. baumannii.

All bacterial cells, including gram-positive and gramnegative bacteria, produce extracellular vesicles (EVs) [10-12]. Bacteria-derived EVs are involved in biological processes such as nutrient acquisition, biofilm formation, horizontal gene transfer, and cell to cell communication [10, 13-18]. Also, bacterial EVs contribute to pathogenic events in host-pathogen interactions regarding the delivery of virulence factors and toxins, host cell death, and inflammatory responses [13, 19-21]. Little is known about mechanisms of EV biogenesis for gram-positive bacteria, whereas several models for outer membrane vesicle (OMV) biogenesis in gram-negative bacteria have been proposed, including a reduction in crosslinking between peptidoglycans and the outer membrane [21-24], deacylation of lipopolysaccharides [25], accumulation of phospholipids in the outer leaflet of outer membranes [18, 26], and localized membrane remodeling [27], suggesting that OMVs are likely to be produced by several pathways in gram-negative bacteria. OMV production is stimulated by harsh environments, such as presence of antimicrobial agents, and envelope and oxidative stresses [13, 14, 28]. Further, sequestration of divalent cations such as $\mathrm{Mg}^{2+}$ and $\mathrm{Ca}^{2+}$ increases OMV production [18]. However, the effect of $\mathrm{Zn}$ or Zur-regulated genes on OMV biogenesis has not been determined.

In this study, we explored the hypothesis that ZrlA plays a role in bacterial morphogenesis and OMV biogenesis, because ZrlA possesses peptidase activity for peptidoglycan remodeling [8], which may affect the crosslinking between peptidoglycans and outer membranes. Moreover, we further characterized the $z r l A$ gene and its encoded protein, even though ZrlA is known to be a $\mathrm{Zn}$-binding peptidase located in the inner membrane [8].

\section{Results}

Characterization of the zrlA gene and its encoded protein The complete sequence of the $z r l A$ gene and surrounding genes in A. baumannii ATCC 17978 was analyzed (GenBank accession number NZ_CP018664.1). The $z r l A$ gene (A1S_3412) is $684 \mathrm{bp}$ long, and it is predicted to encode 227-amino acid protein. Two adjacent genes, yjiA (A1S_3411) and aroP (A1S_3413), were predicted to encode a putative GTPase and an APC family aromatic amino acid transporter, respectively (Fig. 1a). Sequence analysis showed a palindromic Zur box sequence in the promoter region of the $z r l A$ gene. The $z r l A$ gene was predicted to encode a peptidase of the M15 family (https://www.ebi.ac.uk/merops/), with an 86 residue peptidase domain between amino acids 129-214 (Fig. 1b). In enterococci, VanX is known to be a Zn-dependent Dalanine-D-alanine carboxypeptidase (DD-CPase) with H116, D123, and H184 being Zn-coordinated residues [29]. Three metal ligands (H150, D157, and H204) and an active site residue (Q202) were also present in the peptidase domain of ZrlA. These motifs, HXXXXXXD and QXH, were similar to the motifs HXXXXXXD and WXH found in peptidoglycan hydrolase of Burkholderia pseudomallei phage ST79 [30]. Sequence analysis indicated that the $z r l A$ gene was conserved in all sequenced A. baumannii strains with $97-100 \%$ homology (https:// blast.ncbi.nlm.nih.gov/). All amino acid variations were located outside of peptidase domains. Moreover, the $z r l A$ gene was amplified in all tested clinical A. baumannii isolates from Korean hospitals, as well as in ATCC $19606^{\mathrm{T}}$ (Supplementary Fig. 1). Next, a recombinant ZrlA protein was expressed in Escherichia coli BL21 (Supplementary Fig. 2), and the enzymatic activity of recombinant ZrlA protein was assessed by the fluorimetric $o$-phthaldialdehyde (OPTA) method. The recombinant protein specifically cleaved the terminal D-alanine of $\mathrm{N}_{\alpha}$, $\mathrm{N}_{\varepsilon}$-diacetyl-L-Lys-D-Ala-D-Ala, but its enzymatic activity was lower for acetyl-L-Lys-D-Ala-D-Ala and D-AlaD-Ala (Table 1), suggesting specific DD-CPase activity.

\section{Role of ZrIA in bacterial morphology and antimicrobial susceptibility}

We investigated morphological differences between wild-type and $\triangle z r l A$ mutant OH743 strains because DDCPases contribute to cell separation and peptidoglycan remodeling [31]. The wild-type and $\Delta z r l A$ mutant strains appeared as gram-negative coccobacilli at midexponential phase and no morphological difference was observed between the wild-type and OH743 strains at mid-exponential phase (Fig. 2a). The wild-type strain showed more morphological heterogeneity than the $\triangle z r l A$ mutant strain at stationary phase. Bacterial morphology of the $z r l A$-complemented OH810 strain was partially restored compared with that of the wild- 


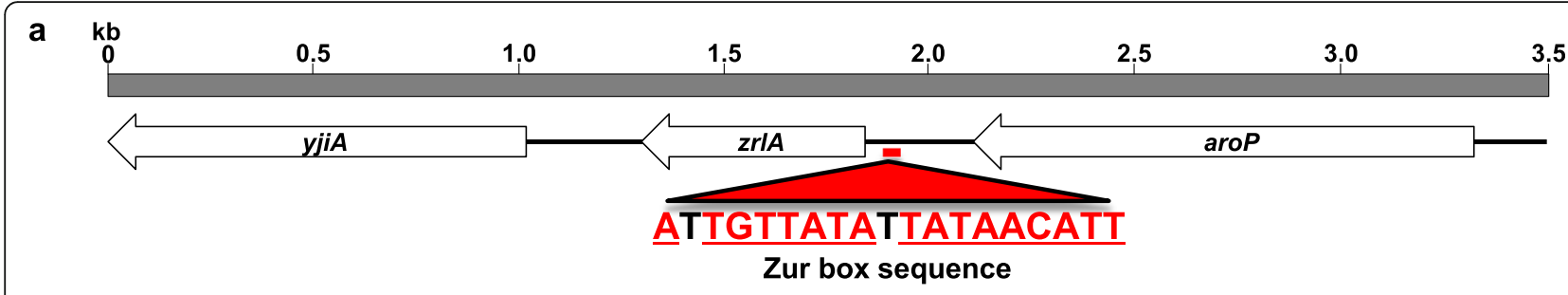

b

$\begin{aligned} & 1 \text { MKRYLGLLVLSLVACSTQTPQPTITHRSPSLPQPSKTVVKIKQQPEDYIVWIATADHRQS } 60 \\ & 61 \text { VQAYKQFLKQKGLADLVPDHELLRSARDWQKCGVEPYAVPPREIWSNIVPTLNILKALVD } 120 \\ & 121 \text { DGVINDFEVTSVYRALALNRCAGGADASRHVFNAALDFRIGPEQPSDLDQFNIQQTKTKL } 180 \\ & 181 \text { CQFWATKGQAFNMGLGVYASGQIHIDSQGFRAWGPDHHYRTSICQGL } 227\end{aligned}$

Fig. 1 Physical map and sequence analysis of the zrlA gene in A. baumannii ATCC 17978. a Arrows indicate coding regions of yjiA, zrlA, and aroP. The figure was generated from the nucleotide sequence of A. baumannii (GenBank Accession No. NZ_CP018664.1). Predicted Zur box sequences are indicated in red. $b$ Analysis of the predicted amino acid sequence of ZrlA using the MEROPS database (https://www.ebi.ac.uk/merops/). The peptidase domain is indicated with solid underlining. The active site (red) and metal ion ligands (blue) are also indicated

type strain. Expression of the $z r l A$ gene in A. baumannii ATCC 17978 was higher during stationary phase than during exponential phase (Fig. 2b). Minimum inhibitory concentrations (MICs) of 15 antimicrobial agents for the wild-type, $\mathrm{OH} 743$, and $\mathrm{OH} 810$ strains were determined to address the effects of $\triangle z r l A$ mutation on antimicrobial susceptibility. Gentamicin showed a 4-fold decrease in MICs for the OH743 strain, and colistin, tobramycin, and erythromycin showed a 2-fold decrease in MICs for the OH743 strain (Table 2). MICs for the remaining antimicrobial agents for the OH743 strain were the same or similar $(<2$-fold change) to MICs for the wild-type strain. These results suggest that ZrlA contributes to bacterial morphogenesis and moderate resistance to several antimicrobial agents.

\section{Role of zrIA in the production of OMVs}

Bacteria were cultured in lysogeny broth (LB) and OMVs were isolated from culture supernatants. Sizes of OMVs from the wild-type, OH743, and OH810 strains were $197.8 \pm 16.0 \mathrm{~nm}, 180.9 \pm 25.9 \mathrm{~nm}$, and $190.7 \pm 18.2$ $\mathrm{nm}$, respectively, using nanoparticle tracking analysis (NTA) (Fig. 3a). OMV particles from $1 \mathrm{~L}$ culture of the

Table 1 DD-CPase activity of the recombinant ZrlA protein

\begin{tabular}{ll}
\hline Substrate & $\begin{array}{l}\text { Enzymatic activity of } \mathbf{r Z r l A} \\
\text { protein }(\mathbf{U} / \mathbf{m g})^{\mathbf{a}}\end{array}$ \\
\hline D-Ala-D-Ala & $9.6 \pm 4.4$ \\
Acetyl-L-Lys-D-Ala-D-Ala & $6.5 \pm 4.2$ \\
$\mathrm{~N}_{a}, \mathrm{~N}_{\varepsilon}$-diacetyl-L-Lys-D-Ala-D-Ala & $34.9 \pm 3.8$
\end{tabular}

${ }^{a}$ Enzymatic activity (U/mg protein) of recombinant ZrlA proteins was determined using the fluorimetric $o$-phthaldialdehyde method. Recombinant ZrlA protein $(40 \mu \mathrm{g})$ was added to $10 \mathrm{mM}$ solutions of indicated substrates.

The results are the mean \pm SD. Assays were performed in triplicate wild-type, $\mathrm{OH} 743$, and $\mathrm{OH} 810$ strains contained $2.96 \times$ $10^{12}, 2.87 \times 10^{13}$, and $2.58 \times 10^{12}$ particles, respectively. The OH743 strain produced more OMV proteins $(457.1 \pm 10.5 \mu \mathrm{g} / \mathrm{L})$ than the wild-type strain $(52.1 \pm$ $4.6 \mu \mathrm{g} / \mathrm{L}$ ) (Fig. 3b). The OH743 strain produced 9.7 times more OMV particles and 8.8 times more OMV proteins than the wild-type strain, but the OH743 strain produced small sizes of OMVs as compared to the wildtype strain. Sodium-dodecyl sulfate-polyacrylamide gel electrophoresis (SDS-PAGE) analysis showed similar protein profiles among the three OMV samples (Fig. 3c). These results suggest that ZrlA negatively affects OMV production in A. baumannii.

\section{Effect of zrIA on OMV-mediated cytotoxicity in epithelial cells}

A549 cells were incubated with A. baumannii OMVs for $24 \mathrm{~h}$ and then cell viability was determined using the 3[4,5-dimethylthiazol-2-yl]-2,5 diphenyltetrazolium bromide (MTT) assay. The wild-type strain OMVs triggered cytotoxicity at $5 \mu \mathrm{g} / \mathrm{ml}$, whereas the $\Delta z r l A$ mutant OMVs triggered cytotoxicity at $\leq 0.625 \mu \mathrm{g} / \mathrm{ml}$ (Fig. 4). Cytotoxicity was significantly different between wildtype and OH743 mutant OMVs at concentrations $\geq 0.625 \mu \mathrm{g} / \mathrm{ml}$. These results suggest that the $z r l A$ gene negatively affects host cell cytotoxicity induced by $A$. baumannii OMVs.

\section{Discussion}

The zrlA gene is known to encode a Zn-binding DDCPase located in the inner membrane [8]. Still, its presence and genetic variability among $A$. baumannii strains are not yet characterized. In silico and polymerase chain reaction (PCR) analyses indicated that the $z r l A$ gene was 


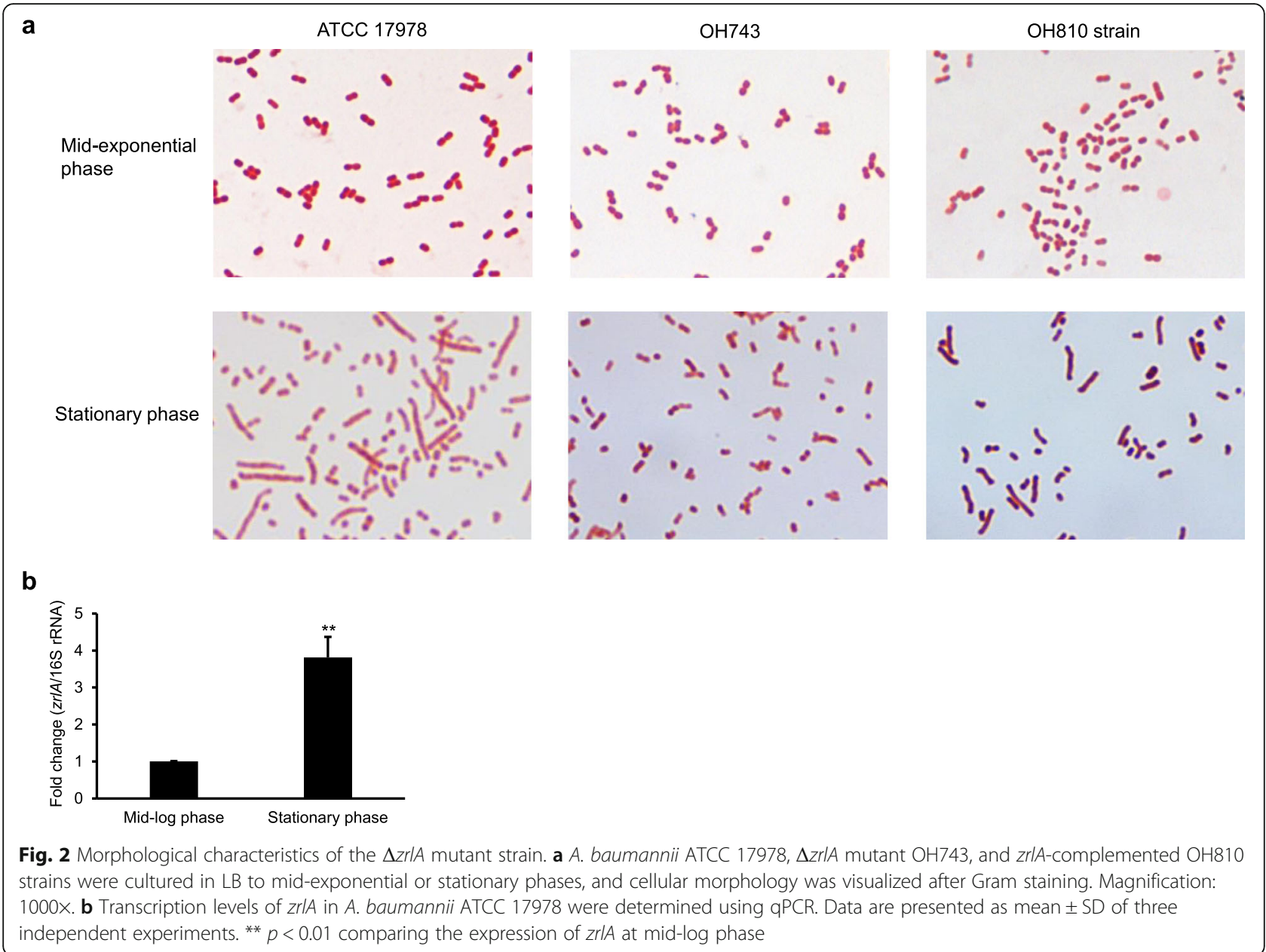

conserved among A. baumannii strains with high sequence homology. Peptidase motifs typically found in DD-CPases were found in the predicted protein encoded by the $z r l A$ gene. Recombinant ZrlA proteins specifically cleaved the terminal D-alanine of $\mathrm{N}_{\alpha}, \mathrm{N}_{\varepsilon}$-diacetyl-L-LysD-Ala-D-Ala, a substrate for penicillin-sensitive Dalanine CPase. Zur-regulated $z r l A$ gene is thus conserved in A. baumannii strains, and its encoding protein ZrlA possesses a specific DD-CPase activity.

DD-CPase, a member of the penicillin-binding proteins (PBPs), cleaves the terminal D-alanine from a muramyl pentapeptide. PBPs are classified into high molecular mass (HMM) and low molecular mass (LMM) PBPs based on amino acid sequence, molecular weight, and enzymatic activity [31-33]. HMM PBPs are responsible for polymerization of peptidoglycan and interstrand crosslinking of adjacent peptidoglycan molecules [31, 34, 35]. HMM PBPs play a role in bacterial cell elongation, maintenance of cell morphology, and cell division. LMM PBPs, including DD-CPases and endopeptidases, contribute to cell separation and peptidoglycan remodeling. LMM PBPs are not essential for bacterial growth. The structure and function of HMM PBPs are well studied, but biological functions of LMM PBPs remain poorly investigated. In limited studies of $A$. baumannii, a LMM PBP $7 / 8$, a D-alanine-D-alanine endopeptidase, was critical for survival in vitro and in vivo and contributed to serum resistance [36]. A deletion mutant of the PBP $7 / 8$ gene in A. baumannii showed more coccobacillary forms than wild-type. Deletion of PBP7/8 seems to alter the structure of peptidoglycan, which in turn affects cell morphology and survival of $A$. baumannii both in vitro and in vivo. In the previous study, bacterial growth was not different between wild-type and $\triangle z r l A$ mutant strains cultured under shaking and static conditions [9], indicating that the $z r l A$ gene is not essential for bacterial survival and growth. However, in the present study, wild-type A. baumannii grown to stationary phase showed more morphological heterogeneity than the $\Delta z r l A$ mutant strain. Morphological difference was not observed between wild-type and $\triangle z r l A$ mutant strains at mid-exponential phase, in contrast to a previous study that demonstrated more morphological heterogeneity in wild-type than 
Table 2 MICs of antimicrobial agents for A. baumannii strains used in this study

\begin{tabular}{llll}
\hline Antimicrobial agent & MIC $(\boldsymbol{\mu g} / \mathbf{m l})$ & & \\
\cline { 2 - 4 } & ATCC 17978 & OH743 & OH810 \\
\hline Aztreonam $^{\mathrm{a}}$ & 24 & 24 & 24 \\
Ceftazidime $^{\mathrm{a}}$ & 3 & 3 & 3 \\
Cefoxitin $^{\mathrm{b}}$ & 128 & 128 & 128 \\
Imipenem $^{\mathrm{a}}$ & 0.19 & 0.19 & 0.19 \\
Meropenem $^{\mathrm{b}}$ & 0.5 & 0.5 & 0.5 \\
Colistin $^{\mathrm{b}}$ & 0.25 & 0.125 & 0.25 \\
Ciprofloxacin $^{\mathrm{a}}$ & 0.125 & 0.19 & 0.125 \\
Levofloxacin $^{\mathrm{b}}$ & 0.125 & 0.125 & 0.125 \\
Nalidixic acid $^{\mathrm{a}}$ & 4 & 3 & 3 \\
Gentamicin $^{\mathrm{a}}$ & 0.5 & 0.125 & 0.25 \\
Tobramycin $^{\mathrm{b}}$ & 0.5 & 0.25 & 0.5 \\
Tetracycline $^{\mathrm{a}}$ & 1.5 & 1.5 & 1.5 \\
Tigecycline $^{\mathrm{b}}$ & 0.125 & 0.094 & 0.125 \\
Trimethoprim $^{\mathrm{a}}$ & $>32$ & $>32$ & $>32$ \\
Erythromycin $^{\mathrm{b}}$ & 16 & 8 & 16 \\
\hline T $^{\text {The Mics wer }}$ & & &
\end{tabular}

${ }^{a}$ The MICs were determined by the Etest method

${ }^{b}$ The MICs were determined by broth microdilution

$\triangle z r l A$ mutant strains cultured to mid- to lateexponential phase under $\mathrm{Zn}$-replete conditions [8]. The expression of the $z r l A$ gene was higher in sessile cells than planktonic cells [9]. Moreover, morphological heterogeneity is correlated with expression level of the $z r l A$ gene among growth phases. ZrlA contributes to overcoming sub-MICs of antibiotic exposure in vitro and in vivo [8]. The $\Delta z r l A$ mutant exhibits increased envelope permeability. Mutant cells show increased susceptibility to antibiotics, including carbenicillin, vancomycin, tetracycline, and polymyxins B, and detergents, including SDS and ethylenediaminetetraacetic acid, in vitro compared to the wild-type strain [8]. The present study also showed that the $\triangle z r l A$ mutant was more susceptible to gentamicin, colistin, tobramycin, and erythromycin than the wild-type strain, but MICs were not greatly different between the two strains. Our results suggest that ZrlA is an LMM PBP possessing a specific DD-CPase activity involved in morphological plasticity and moderate antimicrobial resistance.

The production of OMVs increases in response to nutrient restriction and exposure to antibiotics or chemicals $[13,14,28]$. A reduction in crosslinking between the outer membrane and peptidoglycan also increases OMV production [21-24]. OmpA via the C-terminal OmpAlike domain interacts with diaminopimelate of peptidoglycans [37] and a $\triangle o m p A$ mutant of A. baumannii produced more OMVs than the wild-type strain [38]. In $E$. coli, peptidoglycans are covalently crosslinked to the outer membrane via short outer membrane-anchored lipoprotein Lpp. Levels of Lpp crosslinking to peptidoglycan negatively correlate with OMV production [23, 39]. Spr is a murein DD-endopeptidase located in the outer membrane of $E$. coli. $\Delta s p r$ mutants inhibit peptidoglycan turnover and other PBPs, such as PBP4, induce compensatory increases in peptidoglycan synthesis. This increase of peptidoglycans reduces the ability to form sufficient Lpp-outer membrane crosslinks [40]. This mutation may result in four times more OMV production in $\Delta s p r$ mutant than wild-type. The equilibrium between peptidoglycan synthesis and degradation may affect OMV production by altering the numbers of covalently crosslinked Lpp to peptidoglycan. In the present study, the $\triangle z r l A$ mutant produced 9.7-fold more OMV particles than the wild-type strain. However, sizes of OMVs from the $\Delta z r l A$ mutant were smaller than those from the wildtype strain. Regarding the number of OMV particles and their protein concentrations, each OMV particle from the $\triangle z r l A$ mutant strain may carry slightly less proteins than OMVs from the wild-type strain. ZrlA in A. baumannii displays peptidase activity like $\mathrm{Spr}$ in $E$. coli. Hence, the $\triangle z r l A$ mutant may inhibit peptidoglycan remodeling and decrease interactions between outer membrane proteins and peptidoglycans, resulting in increased envelope permeability and hyperproduction of OMVs.

Stress-inducing conditions alter both production and molecular composition of OMVs [28, 38]. Alterations in lipopolysaccharides, proteins, peptidoglycans, and pathogenassociated molecular patterns of OMVs likely trigger different host cell responses. OMVs from a $\triangle b f m S$ mutant were more cytotoxic in A549 cells than OMVs from the wildtype strain [41], yet OMVs from the $\triangle o m p A$ mutant were less cytotoxic than OMVs from the wild-type strain [19]. The present study showed that OMVs from the $\triangle z r l A$ mutant were more cytotoxic in A549 cells than the wild-type strain. OmpA was identified as a cytotoxic factor packaged in A. baumannii OMVs [19]. SDS-PAGE analysis of OMVs revealed that protein profiles were similar between the wild-type and $\triangle z r l A$ mutant strains. Even though SDSPAGE images show similar profiles, differences in protein content between OMVs from the wild-type and $\triangle z r l A \mathrm{mu}-$ tant strains cannot be excluded. SDS-PAGE analysis is less appropriate than two-dimensional gel electrophoresis. This study did not identify difference in protein content and cytotoxic factors in the $\triangle z r l A$ mutant OMVs, an issue that should be investigated in further studies.

\section{Conclusions}

The present study demonstrates the interplay between ZrlA, peptidoglycan dynamics, bacterial morphogenesis, and OMV production in A. baumannii. ZrlA contributes to overcoming antibiotic exposure and augments pathogenicity of $A$. baumannii both in vitro and in vivo [7-9]. The $z r l A$ gene or its protein is a possible therapeutic 

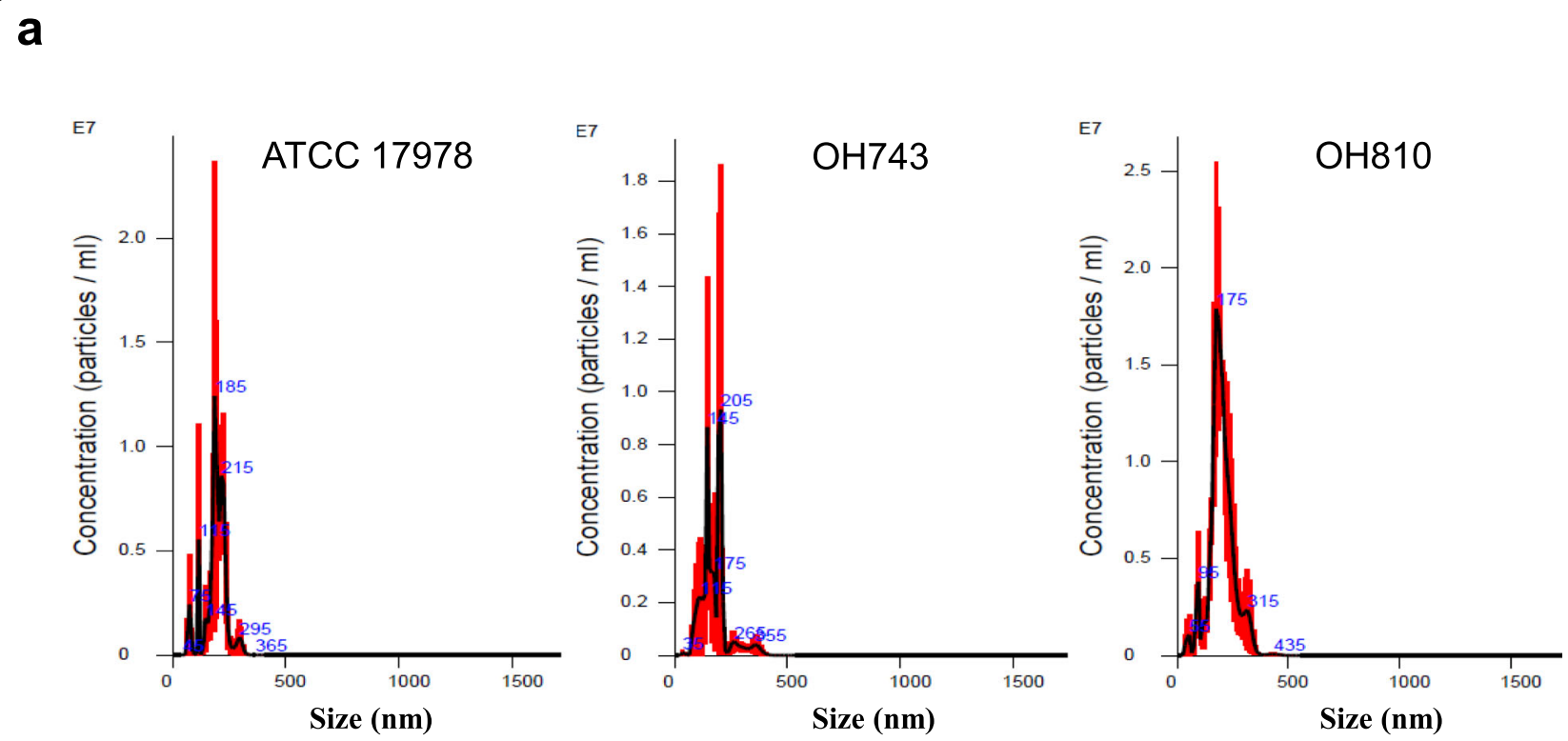

b

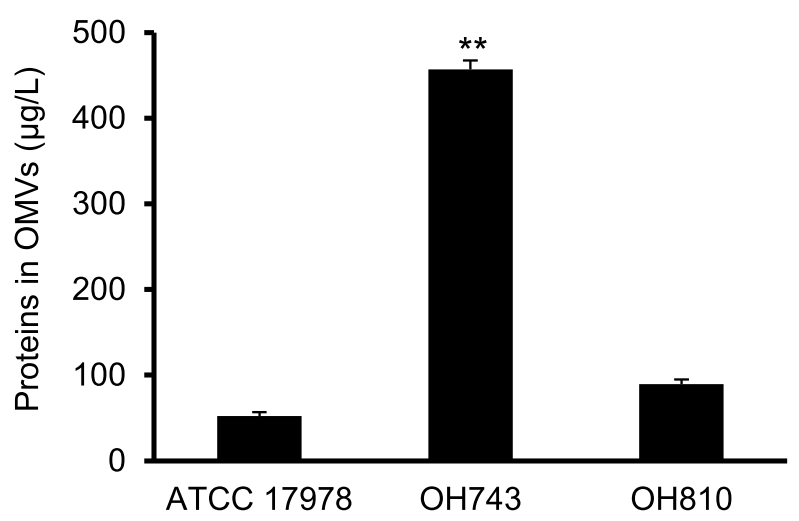

C

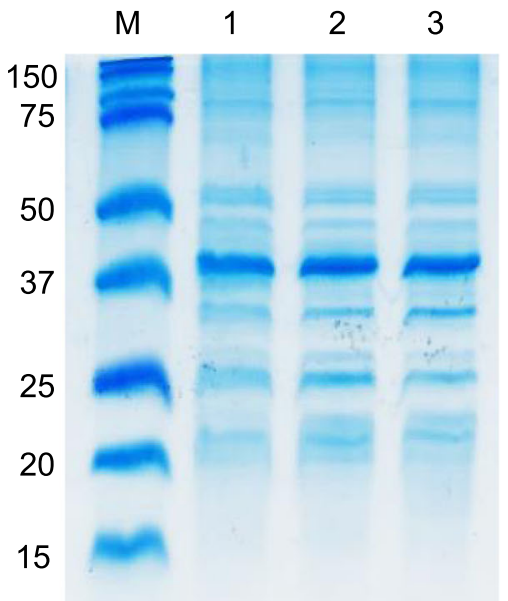

Fig. 3 OMVs of A. baumannii ATCC 17978, $\triangle$ zrlA mutant OH743, and zrlA-complemented OH810 strains a Size and number of OMVs from A. baumannii strains were determined using NTA. Data are representative of three independent experiments with similar results. $\mathbf{b}$ Protein concentration of OMVs isolated from $1 \mathrm{~L}$ of bacterial culture was measured. Data are presented as mean $\pm \mathrm{SD}$ of two independent experiments. ** $p<0.01$ compared to wild-type ATCC 17978. c OMV proteins were resolved by SDS-PAGE in 12\% gels. Lane M, molecular weight marker; 1, A. baumannii ATCC 17978; 2, $\Delta$ zrIA mutant OH743; 3, $\Delta$ zrIA-complemented OH810

target for treating A. baumannii infection. However, deletion of the $z r l A$ gene increased the OMV production in A. baumannii. Moreover, OMVs produced by the $\triangle z r l A$ mutant were more cytotoxic to epithelial cells than OMVs from the wild-type strain. These observations provide opposing perspectives of ZrlA for antivirulence strategies against $A$. baumannii [42].

\section{Methods}

\section{Bacterial strains}

A. baumannii ATCC 17978, $\Delta z r l A$ mutant OH743 strain (ATCC 17978 with $\triangle z r l A$ ), and $z r l A$-complemented
OH810 strain (OH743 with $z r l A$ in chromosome) were used in this study [9]. Ten clinical A. baumannii isolates were obtained from the Kyungpook National University Hospital Culture Collection for Pathogens (KNUHCCP). E. coli BL21 (DE3) star cells were used for production of recombinant ZrlA proteins. Bacteria were cultured in LB or blood agar plates at $37^{\circ} \mathrm{C}$.

Detection and cloning of the $z r l A$ gene and recombinant protein production

PCR was performed to detect the $z r l A$ gene using primers A1 (5' -GCT TTT ATA GTC CCT GAC A-3') 


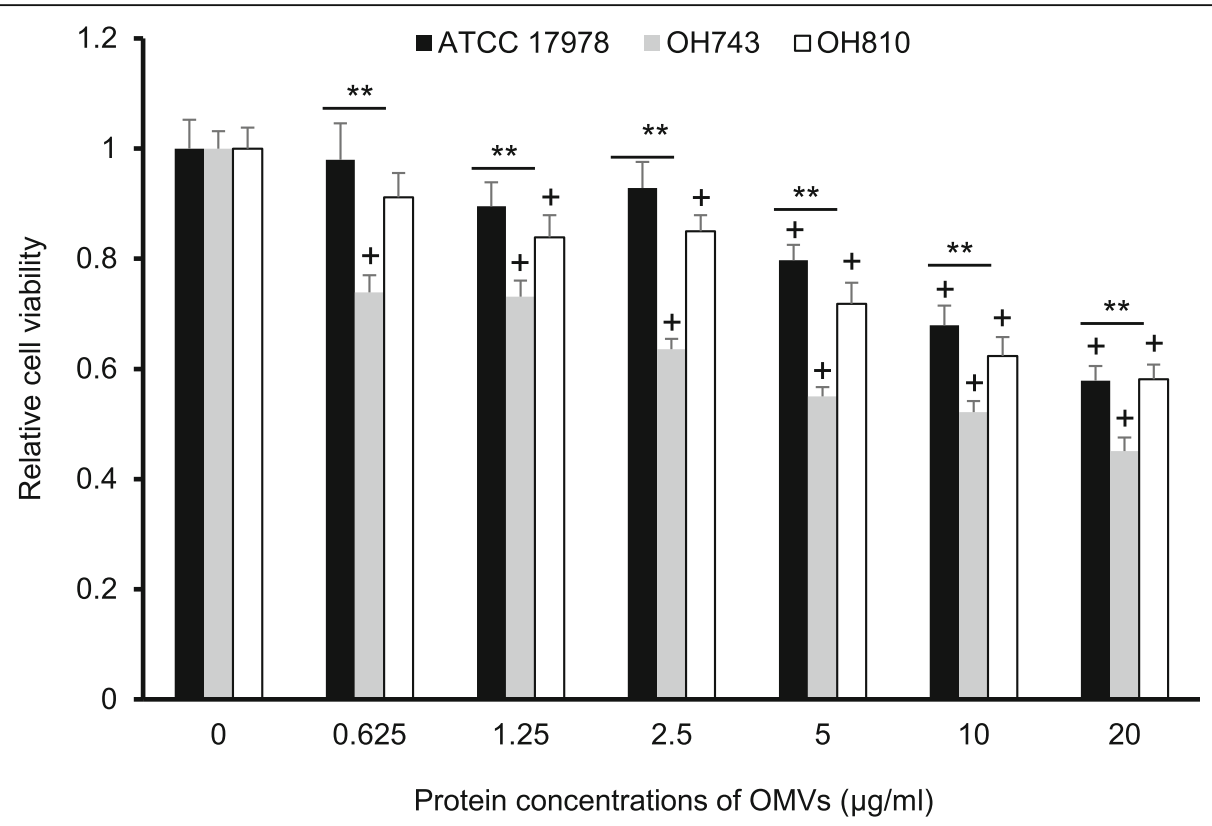

Fig. 4 Host cell cytotoxicity induced by A. baumannii OMVs. A549 cells were treated with OMVs from A. baumannii strains for $24 \mathrm{~h}$ and then cell viability was determined using the MTT assay. Data are presented as mean \pm SD of three independent experiments. $+p<0.01$ compared to untreated control cells. ** $p<0.01$ comparing the same concentration of OMVs from A. baumannii ATCC 17978

and A2 (5'-CTG TGG TTA AAA TCA AAC AA-3'). Genomic DNA was purified from A. baumannii strains using the SolGent ${ }^{\mathrm{tm}}$ Genomic DNA prep kit (SolGent, Daejeon, Korea). The full-length $z r l A$ gene was amplified using primers $\mathrm{C} 1$ (5'- GGG CGG CGG TGG TGG CGG CAT GAA GCG TTA TTT AGG TTT A-3') and C2 (5' - GTT CTT CTC CTT TGC GCC CTA TAG TCC CTG ACA AAT TGA GG-3'), designed for ligation-independent cloning [43]. A. baumannii ATCC 17978 genomic DNA was used as the PCR template. PCR products were treated with T4 DNA polymerase (New England Biolabs, Ipswich, MA) and inserted into the ligation-independent cloning expression vector $\mathrm{pB} 4$, a derivative of pET21a (Novagen, Madison, WI) [44]. DNA fragments and plasmid DNA were purified using the AccuPrep Gel Purification Kit (Bioneer, Daejeon, Korea) and the AccuPrep Plasmid Extraction Kit (Bioneer), respectively. Plasmid construct pB4:zrlA was transformed into E. coli BL21 (DE3) star cells. ZrlA protein was purified using sequential chromatographic steps as previously described [45].

\section{DD-CPase assays of recombinant ZrlA protein}

Enzyme activity of recombinant ZrlA protein was assessed by measuring the release of D-Ala from $N_{\alpha}, N_{\varepsilon^{-}}$ diacetyl-L-Lys-D-Ala-D-Ala, acetyl-L-Lys-D-Ala-D-Ala, and D-Ala-D-Ala (Sigma-Aldrich, St. Louis, MO) using the OPTA method as previously described [46, 47]. Fluorescence intensity $(\lambda \mathrm{ex}=340 \mathrm{~nm}: \quad \lambda \mathrm{em}=455 \mathrm{~nm})$ was measured using a fluorescence microplate reader
(Tecan Spark 10M, Austria). Enzymatic activity was quantified using a standard curve with D-Ala. One unit of DD-CPase activity was defined as the amount of enzyme that produced $1 \mu \mathrm{M}$ of D-Ala per min [46]. Assays were performed in triplicate.

\section{Gram staining of bacteria}

Bacteria were cultured in LB with shaking to optical density at $600 \mathrm{~nm}\left(\mathrm{OD}_{600}\right)$ of 1.2 (mid-exponential phase) or 1.8 (stationary phase). Bacteria were washed with phosphate-buffered saline (PBS). After centrifugation, bacterial pellets were stained with Gram's reagents.

\section{RNA isolation and quantitative real time PCR}

Bacteria were cultured in LB with shaking to $\mathrm{OD}_{600}$ of 1.2 and 1.8 for mid-exponential and stationary phases, respectively. Total RNA was extracted using a RNeasy Mini Kit (Qiagen, Valencia, CA). cDNA was synthesized by reverse transcription with $1.5 \mu \mathrm{g}$ of total RNA using random hexamer primer and RevertAid reverse transcriptase in a total reaction volume of $20 \mu$ l (Thermo Fisher Scientific, Waltham, MA). Specific primers for the $z r l A$ gene, 5'-CCC AGC CGA CGA TTA CTC AT-3' and 5'-GCG ATC CAA ACG ACA TAA TCT TC-3', and $16 \mathrm{~S}$ rRNA gene, 5'-GCA CAA GCG GTG GAG CAT-3' and 5'-CGA AGG CAC CAA TCC ATC TC3 ', were used. Gene transcripts was quantified using a Thermal Cycler Dice TP850 (Takara Bio, Shiga, Japan) with SYBR Premix Ex Taq (Takara Bio) following the manufacturer's instructions. Amplification specificity 
was evaluated using melting curve analysis. Gene expression was normalized to $16 \mathrm{~S}$ rRNA expression in each sample, and fold change was analyzed using the $\Delta \Delta \mathrm{Ct}$ method.

\section{Antimicrobial susceptibility test}

The MICs of aztreonam, ceftazidime, nalidixic acid, ciprofloxacin, gentamicin, imipenem, tetracycline, and trimethoprim were determined by the Etest method following the manufacturer's instructions (bioMe'rieux, Marcy-l'_Etoile, France). MICs of colistin, tigecycline, cefoxitin, meropenem, levofloxacin, erythromycin, and tobramycin were determined by broth microdilution following guidelines of the Clinical Laboratory Standards Institute (CLSI) [48]. E. coli ATCC 25922 and Pseudomonas aeruginosa ATCC 27853 were used as quality control strains.

\section{Isolation of OMVs}

The OMVs derived from A. baumannii were purified from culture supernatants as previously described [19, 28]. In brief, bacteria were cultured in LB with shaking to reach $\mathrm{OD}_{600}$ of 1.5 . Bacterial cells were removed by centrifugation at $8000 \mathrm{~g}$ for $20 \mathrm{~min}$ and then culture supernatants were filtered with a $0.22 \mu \mathrm{m}$ membrane. The samples were concentrated using a QuixStand Benchtop System (GE Healthcare, Amersham, UK) with a $500 \mathrm{kDa}$ hollow fiber membrane (GE Healthcare). OMVs were isolated by ultracentrifugation at $150,000 \mathrm{~g}$ at $4{ }^{\circ} \mathrm{C}$ for 3 h. OMVs were resuspended in PBS and protein contents were measured with a modified bicinchoninic acid (BCA) assay (Thermo Fisher Scientific). OMV proteins were separated on 12\% SDS-PAGE gel and stained with Coomassie brilliant blue R-250 (Bio-Rad, Hercules, CA). The sterility of OMV samples were checked by streaking on blood agar plates.

\section{Nanoparticle tracking analysis}

Size and number of OMVs were measured by a NanoSight NS500 instrument with $488 \mathrm{~nm}$ laser and sCMOS camera modules (Malvern Instruments, Worcestershire, UK) [49]. Captured data were analyzed using NTA 3.1 software build 3.1.46. Experiments were performed in triplicate.

\section{Cell culture and cell viability test}

A549 cells derived from human lung carcinoma were obtained from the Korean Cell Line Bank (Seoul, Korea). Cells were grown in RPMI 1640 medium (HyClone, Logan, UT) supplemented with $10 \%$ heat inactivated fetal bovine serum (HyClone), $2.0 \mathrm{mM}_{\mathrm{L}}$-glutamine, and 100 $\mathrm{U} / \mathrm{ml}$ penicillin at $37{ }^{\circ} \mathrm{C}$ in a $5 \% \mathrm{CO}_{2}$. Cells were seeded at a concentration of $2 \times 10^{4} /$ well in a 96-well microplate. Cell viability was measured using an MTT assay
(Sigma-Aldrich). A549 cells were treated with A. baumannii OMVs for $24 \mathrm{~h}$ and then viability of cells was determined at $600 \mathrm{~nm} 3 \mathrm{~h}$ after treatment with MTT reagent. Assays were performed in triplicate in three independent experiments.

\section{Statistical analysis}

All data are presented as mean \pm standard deviation (SD). Data were analyzed using R 3.6.3 (https://www.rproject.org/). The statistical significance of difference was calculated using nonparametric one-way ANOVA with Dunnett's post hoc analysis or Student's t-test. $P$ values of $<0.05$ were considered statistically significant.

\section{Supplementary Information}

The online version contains supplementary material available at https://doi. org/10.1186/s12866-020-02083-0.

Additional file 1: Table S1. Supplementary table shows the raw data in the manuscript.

Additional file 2: Figure S1. PCR amplification of the $z r / A$ gene in $A$. baumannii strains. Amplicons of $579 \mathrm{bp}$ were detected in all A. baumannii strains tested. Figure S2. Production of recombinant ZrlA proteins. SDSPAGE was performed to detect recombinant proteins of ca. $24 \mathrm{kDa}$ (arrow).

\section{Abbreviations}

EVs: Extracellular vesicles; OMV: Outer membrane vesicle; Zn: Zinc; Zur: Zn uptake-regulator; ZrIA: Zur-regulated lipoprotein A; DD-Cpases: D-alanine-Dalanine carboxypeptidases; OPTA: Fluorimetric o-phthaldialdehyde; MICs: Minimum inhibitory concentrations; NTA: Nanoparticle tracking analysis; PBPs: Penicillin-binding proteins; HMM: High molecular mass; LMM: Low molecular mass

\section{Acknowledgments}

The abstract of this paper was presented at the e-Conference of 2020 Annual Meeting of the Microbiological Society of Korea, October 7-8, 2020, Gunsan,

Korea, as a poster presentation: http://www.msk.or.kr/msk/2020/2020poster.pdf

\section{Authors' contributions}

Conceived and designed the experiments: MHO, SIK, MS, YCL, JCL; Performed the experiments: NK, HJK, SYK, MHK, JHS; Analyzed the data: MHO, SIK, MS, YCL, JCL; Wrote the paper: NK, HJK, MHO, JCL. All authors read and approved the final manuscript.

\section{Funding}

This work was supported by the National Research Foundation of Korea (NRF) grant funded by the Korea government (NRF-2020R1A2B5B01002228).

The funding agency had no role in the design of the study, collection, analysis or interpretation of data, or in the writing of the manuscript.

Availability of data and materials

All data generated or analyzed during this study are included in this published article and its supplementary information files.

Ethics approval and consent to participate

Not applicable.

Consent for publication

Not applicable.

Competing interests

The authors declare that they have no competing interests. 


\section{Author details}

'Department of Microbiology, School of Medicine, Kyungpook National University, 680 Gukchaebosang-ro, Jung-gu, Daegu 41944, Republic of Korea. ${ }^{2}$ Department of Nanobiomedical Science, Dankook University, Cheonan, South Korea. ${ }^{3}$ Drug \& Disease Target Team, Korea Basic Science Institute, Ochang, South Korea. ${ }^{4}$ Department of Bio-Analytical Science, University of Science and Technology (UST), Daejeon, South Korea.

Received: 10 May 2020 Accepted: 29 December 2020

Published online: 18 January 2021

\section{References}

1. Peleg AY, Seifert $H$, Paterson DL. Acinetobacter baumannii: emergence of a successful pathogen. Clin Microbiol Rev. 2008;21:538-82.

2. Dijkshoorn L, Nemec A, Seifert H. An increasing threat in hospitals: multidrug-resistant Acinetobacter baumannii. Nat Rev Microbiol. 2007;5: 939-51.

3. Pendleton JN, Gorman SP, Gilmore BF. Clinical relevance of the ESKAPE pathogens. Expert Rev Anti-Infect Ther. 2013;11:297-308.

4. Hood MI, Skaar EP. Nutritional immunity: transition metals at the pathogenhost interface. Nat Rev Microbiol. 2012;10:525-37.

5. Juttukonda LJ, Chazin WJ, Skaar EP. Acinetobacter baumannii coordinates urea metabolism with metal import to resist host-mediated metal limitation. mBio. 2016:7:e01475-16.

6. Mortensen BL, Skaar EP. The contribution of nutrient metal acquisition and metabolism to Acinetobacter baumannii survival within the host. Front Cell Infect Microbiol. 2013;3:95.

7. Mortensen BL, Rathi S, Chazin WJ, Skaar EP. Acinetobacter baumannii response to host-mediated zinc limitation requires the transcriptional regulator Zur. J Bacteriol. 2014;196:2616-26.

8. Lonergan ZR, Nairn BL, Wang J, Hsu YP, Hesse LE, Beavers WN, et al. An Acinetobacter baumannii, zinc-regulated peptidase maintains cell wall integrity during immune-mediated nutrient sequestration. Cell Rep. 2019;26: 2009-18.

9. Lee EK, Choi $\mathrm{CH}$, Oh MH. Zur-regulated lipoprotein a contributes to the fitness of Acinetobacter baumannii. J Microbiol. 2020;58:67-77.

10. Kim JH, Lee J, Park J, Gho YS. Gram-negative and gram-positive bacterial extracellular vesicles. Semin Cell Dev Biol. 2015;40:97-104.

11. Lee EY, Choi DY, Kim DK, Kim JW, Park JO, Kim S, et al. Gram-positive bacteria produce membrane vesicles: proteomics-based characterization of Staphylococcus aureus-derived membrane vesicles. Proteomics. 2009;9: 5425-36.

12. Knox KW, Vesk M, Work E. Relation between excreted lipopolysaccharide complexes and surface structures of a lysine-limited culture of Escherichia coli. J Bacteriol. 1966;92:1206-17.

13. MacDonald IA, Kuehn MJ. Offense and defense: microbial membrane vesicles play both ways. Res Microbiol. 2012;163:607-18.

14. Kulp A, Kuehn MJ. Biological functions and biogenesis of secreted bacterial outer membrane vesicles. Annu Rev Microbiol. 2010;64:163-84.

15. Schooling SR, Beveridge TJ. Membrane vesicles: an overlooked component of the matrices of biofilms. J Bacteriol. 2006;188:5945-57.

16. Nevot M, Deroncele V, Messner P, Guinea J, Mercade E. Characterization of outer membrane vesicles released by the psychrotolerant bacterium Pseudoalteromonas antarctica NF3. Environ Microbiol. 2006;8:1523-33.

17. Domingues $S$, Nielsen KM. Membrane vesicles and horizontal gene transfer in prokaryotes. Curr Opin Microbiol. 2017;38:16-21.

18. Mashburn LM, Whiteley M. Membrane vesicles traffic signals and facilitate group activities in a prokaryote. Nature. 2005;437:422-5.

19. Jin JS, Kwon SO, Moon DC, Gurung M, Lee JH, Kim Sl, et al. Acinetobacter baumannii secretes cytotoxic outer membrane protein a via outer membrane vesicles. PLoS One. 2011;6:e17027.

20. Jun SH, Lee JH, Kim BR, Kim SI, Park TI, Lee JC, et al. Acinetobacter baumannii outer membrane vesicles elicit a potent innate immune response via membrane proteins. PLoS One. 2013;8:e71751.

21. Jan AT. Outer membrane vesicles (OMVs) of gram-negative bacteria: a perspective update. Front Microbiol. 2017;8:1053.

22. Burdett ID, Murray RG. Electron microscope study of septum formation in Escherichia coli strains B and B/r during synchronous growth. J Bacteriol. 1974;119:1039-56.
23. Cascales E, Bernadac A, Gavioli M, Lazzaroni JC, Lloubes R. Pal lipoprotein of Escherichia coli plays a major role in outer membrane integrity. J Bacteriol. 2002;184:754-9.

24. Rolhion N, Barnich N, Claret L, Darfeuille-Michaud A. Strong decrease in invasive ability and outer membrane vesicle release in Crohn's diseaseassociated adherent-invasive Escherichia coli strain LF82 with the $y f g L$ gene deleted. J Bacteriol. 2005;187:2286-96.

25. Elhenawy W, Bording-Jorgensen M, Valguarnera E, Haurat MF, Wine $E_{\text {, }}$ Feldman MF. LPS remodeling triggers formation of outer membrane vesicles in Salmonella. mBio. 2016;7:e00940-16.

26. Roier S, Zingl FG, Cakar F, Durakovic S, Kohl P, Eichmann TO, et al. A novel mechanism for the biogenesis of outer membrane vesicles in gramnegative bacteria. Nat Commun. 2016;7:10515.

27. Schertzer JW, Whiteley M. A bilayer-couple model of bacterial outer membrane vesicle biogenesis. mBio. 2012;3:e00297-11.

28. Yun SH, Park EC, Lee SY, Lee H, Choi CW, Yi YS, et al. Antibiotic treatment modulates protein components of cytotoxic outer membrane vesicles of multidrug-resistant clinical strain, Acinetobacter baumannii DU202. Clin Proteomics. 2018;15:28.

29. McCafferty DG, Lessard IAD, Walsh CT. Mutational analysis of potential zincbinding residues in the active site of the enterococcal D-Ala-D-Ala dipeptidase VanX. Biochemistry. 1997;36:10498-505.

30. Khakhum N, Yordpratum U, Boonmee A, Tattawasart U, Rodrigues JLM, Sermswan RW. Cloning, expression, and characterization of a peptidoglycan hydrolase from the Burkholderia pseudomallei phage ST79. AMB Express. 2016;6:77.

31. Sauvage E, Kerff F, Terrak M, Ayala JA, Charlier P. The penicillin binding proteins: structure and role in peptidoglycan biosynthesis. FEMS Microbiol Rev. 2008;32:234-58

32. Pratt RF. Substrate specificity of bacterial DD-peptidases (penicillin-binding proteins). Cell Mol Life Sci. 2008;65:2138-55.

33. Ogawara H. Penicillin-binding proteins in Actinobacteria. J Antibiot. 2015;68: 223-45.

34. Macheboeuf P, Contreras-Martel C, Job V, Dideberg O, Dessen A. Penicillin binding proteins: key players in bacterial cell cycle and drug resistance processes. FEMS Microbiol Rev. 2006;30:673-91.

35. Vollmer W, Joris $B$, Charlier P, Foster S. Bacterial peptidoglycan (murein) hydrolases. FEMS Microbiol Rev. 2008;32:259286.

36. Russo TA, MacDonald U, Beanan JM, Olson R, MacDonald IJ, Sauberan SL, et al. Penicillin-binding protein $7 / 8$ contributes to the survival of Acinetobacter baumannii in vitro and in vivo. J Infect Dis. 2009;199:513-21.

37. Park JS, Lee WC, Yeo KJ, Ryu KS, Kumarasiri M, Hesek D, et al. Mechanism of anchoring of OmpA protein to the cell wall peptidoglycan of the gramnegative bacterial outer membrane. FASEB J. 2012;26:219-28.

38. Moon DC, Choi CH, Lee JH, Choi CW, Kim HY, Park JS, et al. Acinetobacter baumannii outer membrane protein a modulates the biogenesis of outer membrane vesicles. J Microbiol. 2012;50:155-60.

39. Bernadac A, Gavioli M, Lazzaroni JC, Raina S, Lloubes R. Escherichia coli tolpal mutants form outer membrane vesicles. J Bacteriol. 1998;180:4872-8.

40. Schwechheimer C, Rodriguez DL, Kuehn MJ. Nlpl-mediated modulation of outer membrane vesicle production through peptidoglycan dynamics in Escherichia coli. MicrobiologyOpen. 2015:4:375-89.

41. Kim SY, Kim MH, Kim SI, Son JH, Kim S, Lee YC, et al. The sensor kinase BfmS controls production of outer membrane vesicles in Acinetobacter baumannii. BMC Microbiol. 2019;19:301.

42. Kim HJ, Kim N, Jun SH, Ryu JH, Oh MH, Kim Sl, et al. Contribution of Zurregulated lipoprotein $A$ to bacterial morphogenesis and production of outer membrane vesicles in Acinetobacter baumannii. 2020. http://www. msk.or.kr/msk/2020/2020poster.pdf. Accessed 24 Dec 2020.

43. Aslanidis C, de Jong PJ. Ligation-independent cloning of PCR products (LICPCR). Nucleic Acids Res. 1990;18:6069-74.

44. Sun J, Jeon JH, Shin M, Shin HC, Oh BH, Kim JS. Crystal structure and CRISPR RNA-binding site of the $\mathrm{Cmr} 1$ subunit of the $\mathrm{Cmr}$ interference complex. Acta Crystallogr D Biol Crystallogr. 2014;70(Pt 2):535-43.

45. Kim BN, Shin M, Ha SC, Park SY, Seo PW, Hofmann A, et al. Crystal structure of an ASCH protein from Zymomonas mobilis and its ribonuclease activity specific for single-stranded RNA. Sci Rep. 2017;26:12303.

46. Wright GD, Molinas C, Arthur M, Courvalin P, Walsh CT. Characterization of vanY, a D,D-carboxypeptidase from vancomycin-resistant Enterococcus faecium BM4147. Antimicrob Agents Chemother. 1992;36:1514-8.

47. Binda E, Marcone GL, Berini F, Pollegioni L, Marinelli F. Streptomyces spp. as efficient expression system for a D,D-peptidase/D,D- 
carboxypeptidase involved in glycopeptide antibiotic resistance. BMC Biotechnol. 2013;13:24.

48. Clinical and Laboratory Standards Institute. Performance Standards for Antimicrobial Susceptibility Testing: Twenty-seventh Informational Supplement M100-S28. Wayne: CLSl; 2018.

49. Gerritzen MJH, Martens DE, Wijffels RH, Stork M. High throughput nanoparticle tracking analysis for monitoring outer membrane vesicle production. J Extracell Vesicles. 2017;6:1333883.

\section{Publisher's Note}

Springer Nature remains neutral with regard to jurisdictional claims in published maps and institutional affiliations.

Ready to submit your research? Choose BMC and benefit from:

- fast, convenient online submission

- thorough peer review by experienced researchers in your field

- rapid publication on acceptance

- support for research data, including large and complex data types

- gold Open Access which fosters wider collaboration and increased citations

- maximum visibility for your research: over $100 \mathrm{M}$ website views per year

At BMC, research is always in progress.

Learn more biomedcentral.com/submissions 